\title{
Commentary
}

\section{Cortisol pulsatility and its role in stress regulation and health}

\author{
Elizabeth A. Young ${ }^{\mathrm{a}, \mathrm{b}, *}$, James Abelson ${ }^{\mathrm{a}}$, Stafford L. Lightman ${ }^{\mathrm{c}, 1}$ \\ a Department of Psychiatry, The University of Michigan, USA \\ ${ }^{\mathrm{b}}$ Mental Health Research Institute, The University of Michigan, USA \\ ${ }^{\mathrm{c}}$ Department of Medicine, Bristol Royal Infirmary, Bristol, UK
}

\begin{abstract}
One of the classic characteristics of the human stress response is the wide inter-individual variation. Although there is much current interest in the genetic and environmental contributions to these differences, studies on human subject have been sparse and characterised by methodological problems. The major factor that is rarely taken into account is the intrinsic rhythmicity of hypothalamo-pituitary-adrenal activity, not simply the classic diurnal variation but also the endogenous pulsatility which is similar to, but much less well recognized than, the rhythms found within the reproductive and growth hormone axes. In this review we propose some novel ideas relating to the importance of pulsatility both for the design of human stress-response studies and for their interpretation as well as implications for our understanding of disease.
\end{abstract}

(C) 2004 Elsevier Inc. All rights reserved.

\section{Introduction}

Stressors - whether they be physical or psychological (processive) - activate afferent neural pathways within the central nervous system which project to diencephalic centres where they initiate a response. This response may be behavioral, autonomic and/or endocrine. The principal neuroendocrine system activated in mammals is the hypothalamo-pituitary-adrenal axis, with its 'end' products cortisol (in man) and corticosterone (in rats).

There is a massive literature on stress responses in experimental animals, particularly the rat, and this has allowed us to reach an understanding of some very important principles. (1) There is a very important genetic contribution to the magnitude of the stress response [30]. (2) Early life events can alter programming of the HPA axis to either increase or decrease stress responsive-

\footnotetext{
${ }^{*}$ Corresponding author. Present address: 205 Zina Pitcher Place, Ann Arbor, MI 48109-0720, USA. Fax: +734 6474130.

E-mail address: eayoung@umich.edu (E.A. Young).

${ }^{1}$ Current address: Henry Wellcome Laboratories for Integrative Neuroscience and Endocrinology, University of Bristol, UK.
}

ness [24]. (3) Chronic activation of the neuroendocrine stress response results in changes in hypothalamic regulation of both CRF and AVP mRNA and differential responses to homotypic and heterotypic stressors [7,22]. (4) There is great plasticity of the HPA responses to stressors with reversible changes occurring during pregnancy and lactation [21]. (5) There is considerable inter-individual variability in stress responsiveness even among animals of the same genetic background and the same environmental backgrounds [28].

We have investigated the physiological basis for these heterogeneous responses to stress by using an automated blood sampling system for free-running rats. This has allowed us to make some novel observations about the way in which the HPA is organized. In particular we have been able to demonstrate that the corticosterone is released in a pulsatile manner throughout the $24 \mathrm{~h}$ with a circhoral ultradian rhythm ([28], Fig. 1). Of particular importance is the fact that the release of corticosterone is characterized by alternating periods of HPA activation and inhibition. Indeed during the periods of inhibition (which occur after each endogenous pulse of secretion) there is a refractory period when 


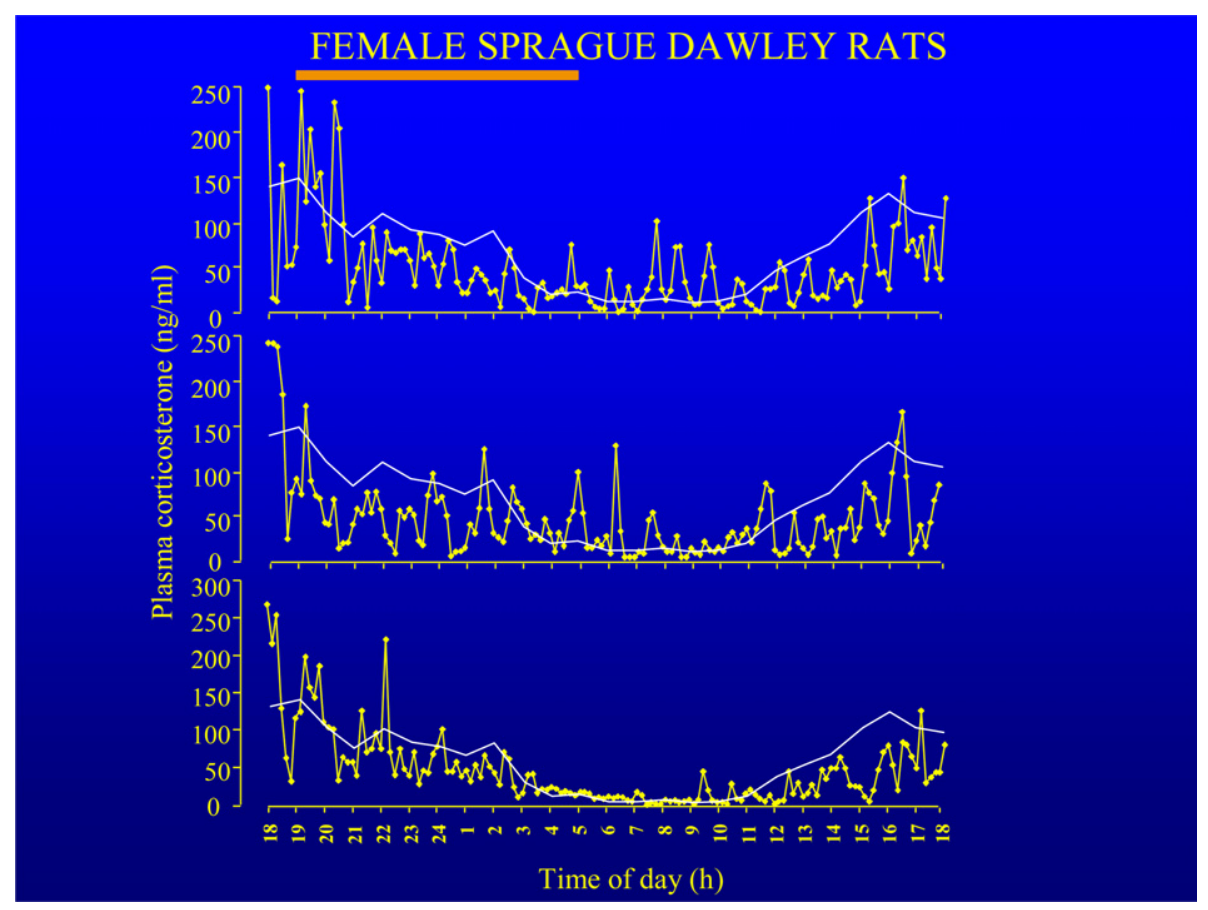

Fig. 1. Twenty-four hour profiles of corticosterone pulses in normal female rats. The individual profiles are graphed along with the mean cortisol across the group. Reprinted with permission from [28].

the HPA is no longer sensitive to activation by a mild stressor (Fig. 2). This of course goes a long way to explain the inter-individual variability in responsiveness as a response to our stress depends on the time during the endogenous secretory cycle that the animal is exposed to the stressor. Furthermore, since the frequency of the pulsatility increases during chronic stress there is an increased proportion of time when the animals

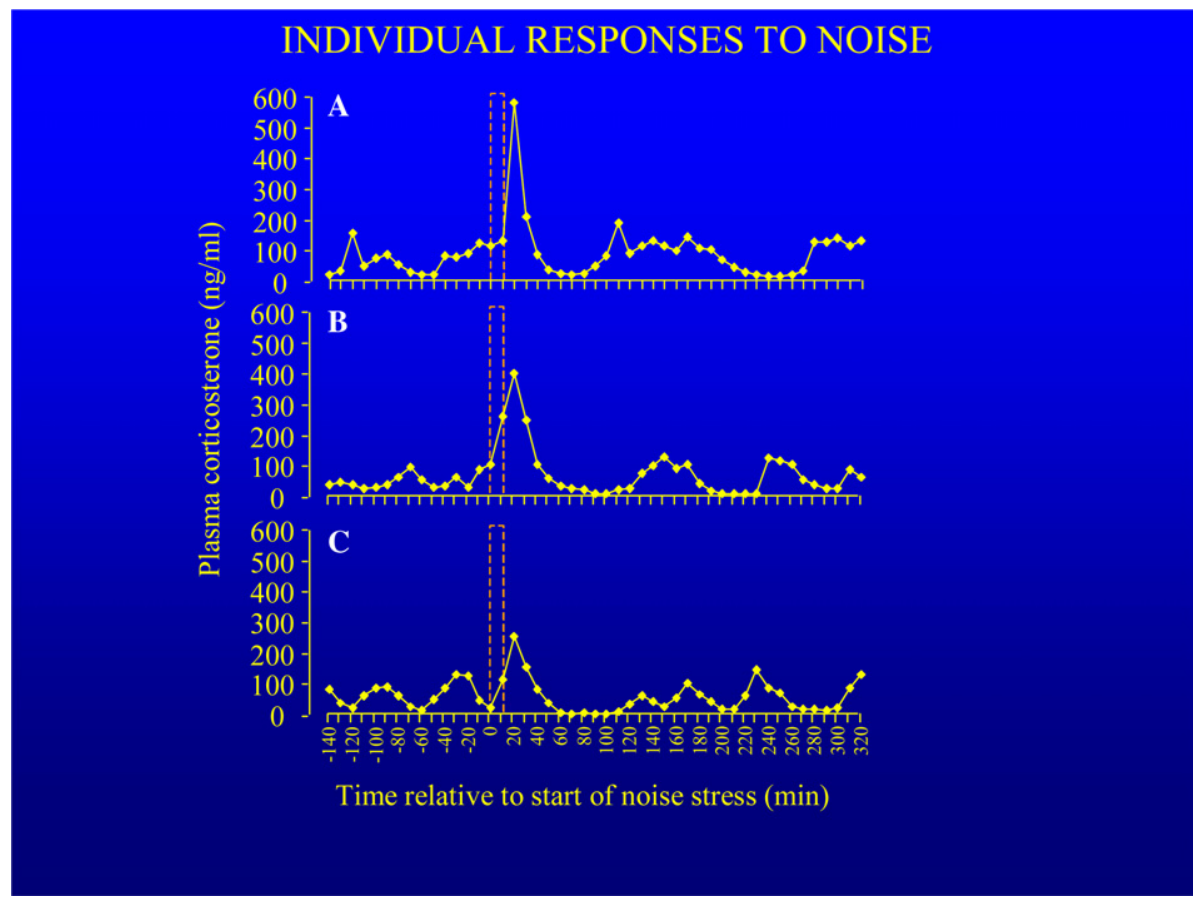

Fig. 2. Panels A-C show individual profiles of corticosterone response in rats given random white noise stressor over the course of the day. In Panels $\mathrm{A}$ and $\mathrm{B}$ the magnitude of the stress response is greater than that of the spontaneous pulses, while this is not the case in Panel C. As can also be seen magnitude of the stress response varies based on phase of the spontaneous pulse in which the stressor is applied (Panels A and B vs Panel C). Reprinted with permission from [28]. 
are in a stress non-responsive state, giving rise to an apparent stress hyporesponsiveness [29].

These data, of course, open up a whole raft of questions about the regulation of the HPA axis in man: the relevance of genetic and environmental factors, the effects of physical and mental disease, and the role of early life events and more recent experiences. Clearly we cannot extrapolate rat data to man, so we need to use these data to inform and design appropriate studies in man. This, however, is far from simple!

One very obvious difference between rats and man is that rats are very sensitive to stress, and a mild stressor will result in a robust increase of corticosterone that approximates to the amplitude of the daily diurnal rhythm (Fig. 2). This of course is very easy to see and quantitate. In man, however, stress responses are much more modest in magnitude and are often very similar to the magnitude of a physiological pulse of cortisol secretion. This fact is frequently forgotten when one looks at group rather than individual responses to a stressor.

Fig. 3 represents of the mean response of human volunteers to a Trier social stress test (TSST, [19]) — a psychological stress evoked by a public speaking task in front of a critical audience. At first sight this seems to be a classic response similar to that which we have seen in the rat. When, however, individual human subject data are examined the stress response becomes much less clear and substantial individual variation becomes apparent. The question we wish to address in this paper is simply this: are the differences in stress responses that can be detected on individual occasions simply a reflection of the psychological complexity of human sensitivities, or do we need a much better understanding of the

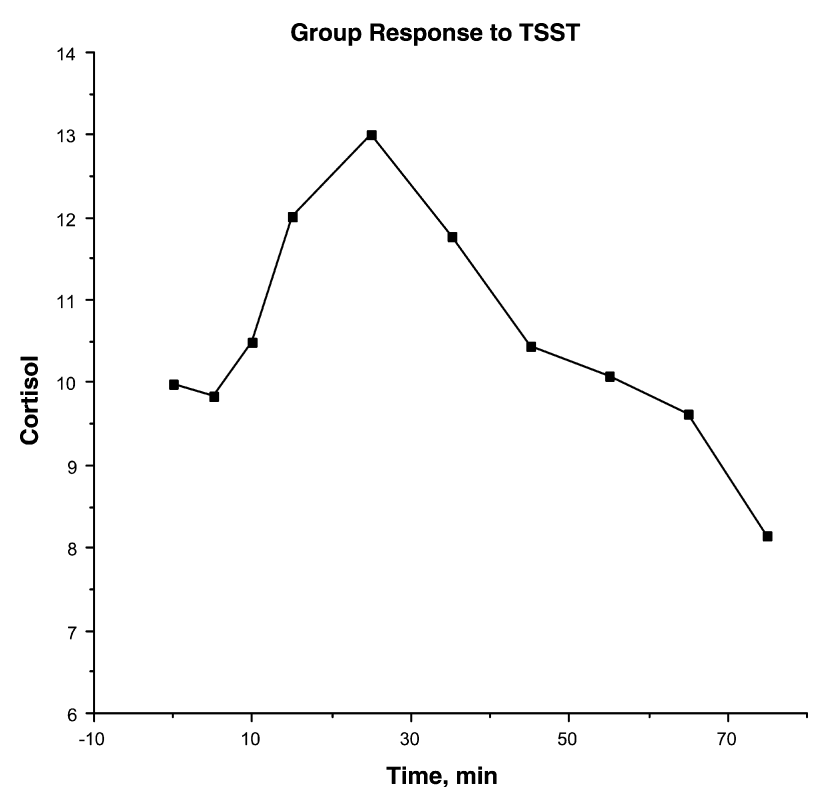

Fig. 3. The mean group cortisol response to a social stressor, the Trier Social stress Test (TSST) in 10 normal subjects. relationship of the modest human stress response to aspects of HPA rhythmicity? What is certainly clear is that our understanding of HPA activity in health and disease in man is remarkably poor when we compare it to our knowledge of the rat, and until this is improved many of our ideas about the interaction of stress and disease will remain in the realm of speculation.

\section{Hormone rhythms and the stress response}

Although the clinical relevance of $\mathrm{GnRH}, \mathrm{LH}$ and FSH pulsatility are well recognized and utilized for therapeutic purposes, the pulsatile nature of cortisol secretion appears to be a rather inconvenient aspect of clinical endocrinology that is usually ignored or assumed to be of no importance. This position is however becoming increasingly untenable as data from the rat demonstrate a critical role for HPA pulsatility in homeostatic regulation. Both rat and man have a well-documented circadian rhythm of corticosterone and cortisol secretion, respectively, and the phase of this rhythm has a significant effect on the response to a stressor [12,20,31]. In the nocturnal rat most studies are conducted in the morning or afternoon, which is the quiescent period of their circadian rhythm, while for similar reasons most human studies are carried out during the afternoon or evening. What has been less appreciated is that both rats and man also have major ultradian rhythms of the HPA axis, which result in a pulsatile release of corticosteroids that continues throughout the 24-h period even during the quiescent phase $[4-7,11,13]$. Thus, if the cortisol patterns of two individuals subjects from the study in Fig. 3 are viewed from a 24-h perspective (Fig. 4, Panels A and B), we see that what looks like a "stress response" in these subjects also looks like the spontaneous pulses that were already occurring before the "stressor" was applied. This is further highlighted by the profiles of subjects in Panels C and D collected under resting conditions, showing spontaneous pulses occurring at the same time. Group data, as shown in Fig. 3, which depicts the mean of 10 subjects undergoing 24-h blood sampling followed by the Trier social stress test, do reveal a stress response in humans; but studying this response is clearly much more difficult when the system's natural rhythms make it difficult to "see" responses at the individual level.

While it might appear that this uncertainty is unique to the response to a psychological stressor, which is less "robust" than endocrine challenges, Fig. 4, Panels E and F shows the data from two subjects undergoing 24-h sampling followed by administration of oCRF, which bypasses the brain and cognitive processing. Again, while in the subject shown in panel $F$ the magnitude of the response to CRF distinguishes it from the sponta- 

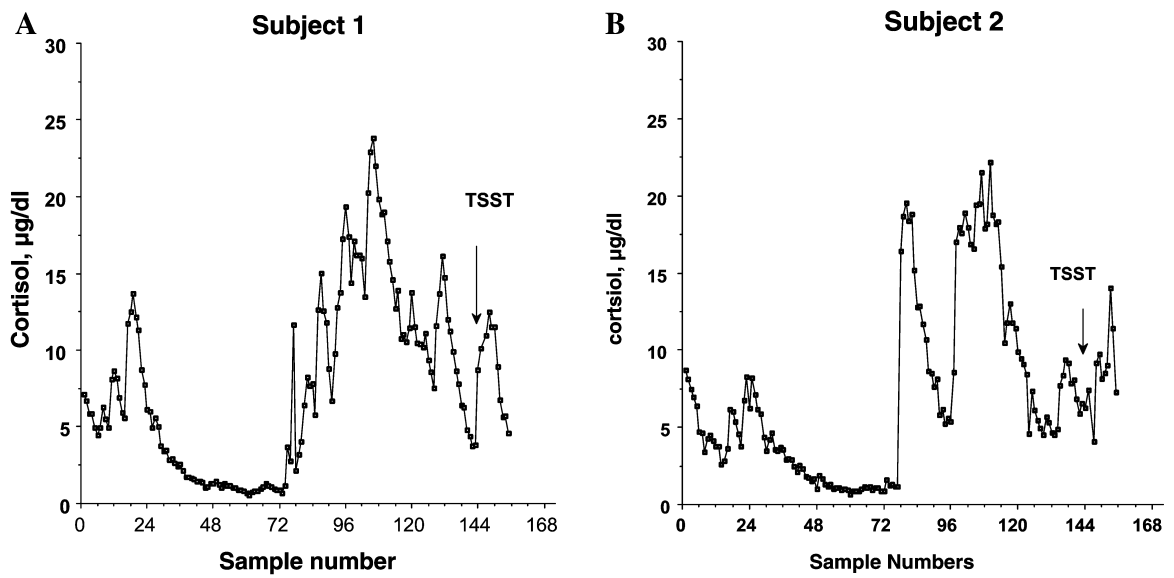

C Normal Subject CM55 Under Basal Conditions

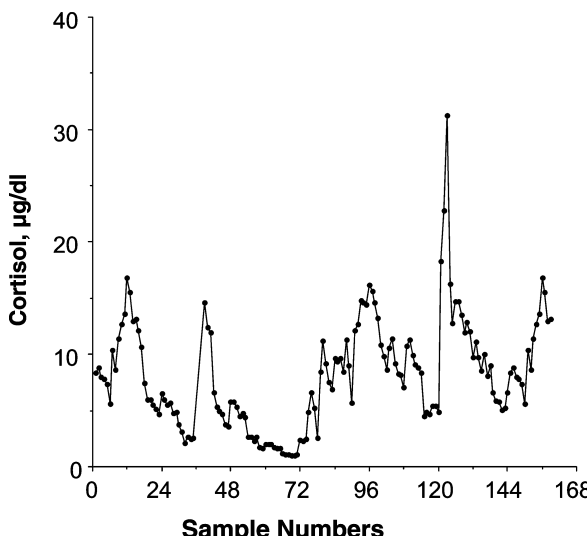

D Normal Control JO75 Under Basal Conditions
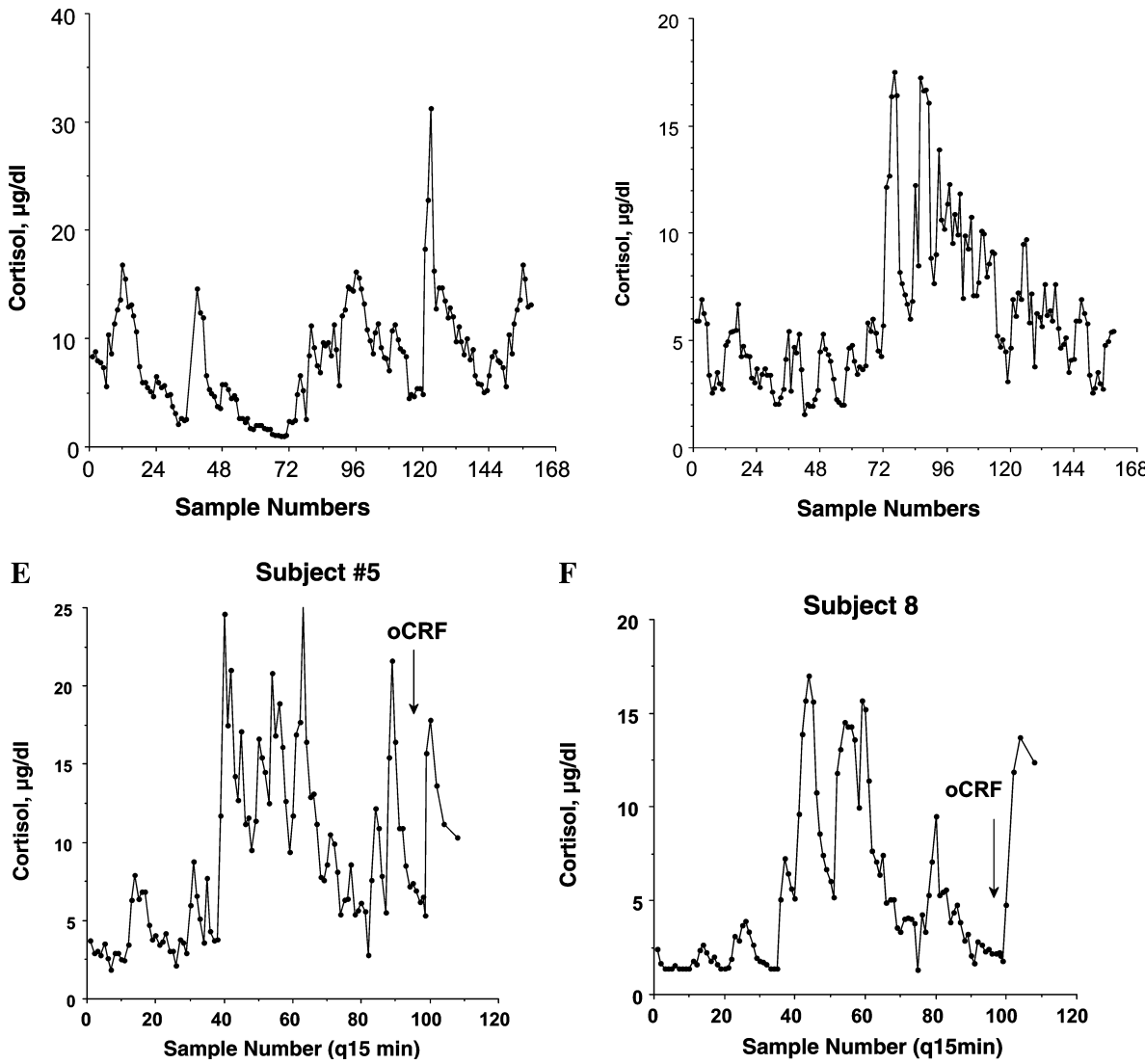

Fig. 4. Twenty-four hour cortisol profile followed by the TSST or oCRF in individual subjects. While both individuals in A and B show some response to the stressor (TSST), when seen in the light of the endogenous cortisol pulses, it is hard to distinguish this activity from an endogenous pulse. This is further highlighted by comparison to profiles of subjects in Panels C and D who had no interventions while undergoing 24-h sampling. In the latter cases, spontaneous pulses occur at similar times to the TSST (sample 144). The same is true for the response to oCRF in panel F, where distinguishing between spontaneous activity and oCRF response is difficult. In contrast, the response to oCRF in panel $\mathrm{E}$ shows a greater magnitude than spontaneous pulses occurring at that phase of the circadian rhythm. Also note that spontaneous pulses of varying amplitude occur throughout the day with clear amplitude decreases late in the evening and overnight in most subjects.

neous pulses occurring in the evening, this is not so in the subject shown in panel E. The circadian rhythm of cortisol secretion becomes even more important, since if CRF were administered during the acrophase (when the natural rhythm is at its peak), even this distinction could be lost. As with the psychological stress data, to clearly "see" the human CRF response is dependent upon "averaging" across peaks and troughs of cortisol secretory episodes to produce a flat baseline, on which the synchronized CRF response can be viewed. 
If a multiple sampling protocol were employed, data similar to that in Figs. 4A-F would be the norm. In most studies, however, sampling is less frequent and conducted over a shorter period of time. As a result, we don't see the underlying pattern of spontaneous pulses, but instead just see tremendous variability in baseline cortisol levels. Furthermore, these spontaneous changes in cortisol, i.e., cortisol pulses, are often viewed as "noise" in the system and dismissed as meaningless fluctuations. Alternatively, investigators may infer causality between other events occurring during the experimental paradigm and the cortisol responses seen, e.g., when the subject was "stressed" by changing rooms or "stressed" upon arrival by the novelty of the situation. In fact, looking at individuals in this short term snapshot of $1-2 \mathrm{~h}$ and viewing these fluctuations as "noise", misses much of the essential information that is communicated by pulsatile cortisol secretion, and therefore misses what is needed to evaluate what the individual and the individual's brain is experiencing during exposure to a stressful stimulus.

\section{Pulsatile secretion: impact and function}

Observing the pulsatile nature of cortisol secretion raises questions about the source of this pulsatile secretion, its mathematical evaluation and the role that it plays in HPA axis regulation. Fourier analysis is often used to study rhythmic fluctuations (oscillatory patterns) - but for non-stationary and non-linear data the basic assumptions of Fourier analysis are no longer valid, and time series need to be decomposed into different modes of oscillation for subsequent quantitative analysis. This type of analysis applied to data from the Lewis rat (Peng, Windle, Ingram and Lightman, unpublished data) shows oscillation on multiple time scales and variation of amplitude and frequency of each oscillation over time. Similarly Young et al. [33] found that time series analysis and pulsatile modeling of cortisol secretion in man showed a very poor fit to observed data unless a circadian variation in baseline secretion was included in the model. In this model the familiar circadian rhythm seen in group 24-h cortisol data can be explained by the circadian baseline with additional small changes in pulse amplitude across the circadian rhythm. It furthermore suggests that pulsatile and circadian baseline components are separable secretory modes and can be independently regulated.

But, is there any function to the cortisol pulsatility? It is well established that pulsatile secretion of GnRH is necessary to prevent receptor desensitization. In fact, the continuous administration of GnRH agonists results in hypogonadism. While continuous administration of CRF results in increased cortisol secretion in humans, this does not necessarily mean that these pulses of corti- sol are not similarly functional in man (or rodents), though it does suggest that the function may well differ from that seen in hypothalamic-pituitary-gonadal system.

Of potential relevance and importance in the HPA axis is the fact that the system is strongly regulated by glucocorticoid negative feedback, which is in turn controlled by two different receptors (MR and GR) with differing affinities. Differing affinities means differential occupation and activation depending on circulating levels of ligand. The relative balance of activation between these two receptors may therefore change rapidly over the course of a cortisol pulse, with the size and spacing of pulses then able to carry regulatory information to the receptors through temporally changing occupation of the two receptor types. Tissue differences in receptor distribution could then allow similar pulses to carry different information to different areas. This model differs from the classic hypothesis regarding glucocorticoid feedback, which suggests that MR plays the predominant regulatory role during the circadian nadir and GR during the circadian peak $[23,25]$. However, studies by Bradbury et al. [4] in rodents and Young et al. [32] in humans have demonstrated that administration of an MR antagonist, spironolactone, results in HPA axis activation in both the peak and the nadir of the circadian rhythm. Similarly, our studies in the rat [3] using a glucocorticoid antagonist suggest a major effect of GR right through the late light phase and into the subsequent $12 \mathrm{~h}$. This indicates that as well as a role of MR throughout the day, GR is also involved even during the late light phase. There is thus a prolonged effect of both MR and GR despite the belief that during peak diurnal secretion cortisol or corticosterone levels are in the GR range overcoming any effect of MR and during the nadir levels should be too low to activate GR.

The concept of rapidly changing concentrations of glucocorticoids has, to our knowledge, never been considered with respect to the pharmacokinetics of receptor occupancy and signal transduction. Our data both in the rat and in man would suggest that over relatively short periods of time there will be widely changing concentrations of ligand available for both MR and GR binding. This is likely to be of particular importance for GR occupancy since MR has such high affinity for corticosterone that short term changes in ligand concentration may only have relatively small effects. GR however has a much lower affinity for corticosterone [8] and it seems very likely that the peaks of the secretory pulse will have a dominant effect on GR occupancy. This may also be tissue-specific depending on access of ligand, and this could be particularly important in the central nervous system from which low levels of cortisol are actually excluded by the P-glycoprotein transporter [18]. Indeed it is quite possible that central nervous system tissues only see the peaks of secretory episodes. The pulses of corti- 
sol could thus affect the ratio of MR:GR occupancy, signal transduction, receptor desensitization, and the recycling of MR and GR - all of which could change during the different phases of the circadian cycle and during stress-induced increases in pulse frequency [27]. It is interesting that the time domain for labeled dexamethasone to be translocated to the nucleus in brains of adrenalectomised rats is about 60-90 $\min$ [9] and interactions between rhythms of ligand presentation and GR activation, translocation and DNA binding may have important transcriptional consequences. In addition to these effects on the classical genomic responses of GR and MR activation, these rapid changes in glucocorticoid levels may activate the non-genomic rapid effect of steroid hormones which are being reported with increasing frequency and which we have found to result in very rapid feedback inhibitory effects on the HPA axis [2,34].

The pulsatile secretion of cortisol does, of course, raise the issue of a "pulse generator". In the classic LH system, the pulse generator has been localized to the arcuate nucleus by the use of multiunit electrical recording. Unfortunately, there has been very little investigation of the putative generator which could regulate the activity of the HPA axis, despite the demonstration of its ultradian as well as its circadian rhythms. It is interesting that both $\mathrm{LH}$ and cortisol pulses have frequencies in the 60-90 min range (circhoral) but this does not imply a common generator, since cortisol pulses and LH pulses are not concordant (Young et al., unpublished data). While the suprachiasmatic nucleus is ultimately the source of the circadian rhythm and synchronization with day night cycles, it is unclear whether it is the paraventricular nucleus of the hypothalamus itself (the location of CRF neuron cell bodies) that is the site of the rhythmic activity of the HPA axis. A recent report has suggested intrinsic rhythmicity of the PVN in vitro [1], and clearly further research needs to be performed to shed light on this issue.

Other possible hormonal influences include phase of the pulse and varying degrees of glucocorticoid negative feedback. In the rat we find that following each peak of corticosterone secretion, there is a refractory period during which the HPA axis is resistant to activation by stressors (Figs. 2B-D) [28]. Thus the timing of a stressor relative to the endogenous HPA pulse cycle likely plays a role in determining the size of the resultant stress response in animal models. It has been much more difficult to investigate such a mechanism in man as we lack short acting, mild stressors that can be used to reproducibly increase HPA activity. In an initial exploration of this issue, we examined the pattern of cortisol secretion for the hour prior to administration of the TSST in 52 subjects. We subdivided the subjects into groups whose cortisol profile prior to stressor exposure was increasing, decreasing, or flat. The mean cortisol responses were not different between groups, and the 18 non-responders were equally distributed between ascending, descending and flat profiles preceding the stressor (Young, unpublished data). This preliminary examination did not support the hypothesis that preceding spontaneous pulses inhibit stress responses in some subjects. However, interpretation of the human data is severely complicated by the reality of anticipation, which is not a factor in animal studies. In the animal models, the onset of the stressor is much more clearly defined, as the animals can be housed in the familiar environments of their home cages and the stressors can be randomly applied, with no opportunity for anticipation. Human subjects must be fully informed about the experiments in which they will participate and they enter into novel environments (laboratory) in order to engage that participation. In this situation it becomes very difficult to define exactly when the "stressor" begins so the temporal dissection of HPA responses is much more difficult. Furthermore, there may be other unidentified environmental factors or cues influencing the response.

\section{Rethinking HPA axis dysregulation and disease}

Considering that both the difficulty in interpreting individual data over a short time span, and the intrinsic pulsatility of the system limits our ability to infer a causal relationship between environmental events and cortisol changes in any one individual, raises the issue of whether it is appropriate to interpret group means as a measure of individual responses. A salient example is aging, where the standard dogma states that there is prolonged stress response, i.e., a failure to "turn off" the cortisol response to stress. Furthermore this prolonged stress response leads to negative consequences on the brain, including hippocampal atrophy. However, if the picture of the stress response to aging is a group picture rather than an individual picture, then an equally likely explanation for the mean data are desynchronization occurring in aging. So some individuals may respond late while others may show a normal stress response. Indeed there is evidence for significant changes in HPA rat rhythmicity with aging both in rats $[5,6,17]$ and man [26]. Using a multiple sampling paradigm examining individual animals over $24 \mathrm{~h}$, we have failed to show any evidence for a prolonged stress response in aging. [17].

"Chronic stress" is another condition that needs to be re-assessed, as it is rarely seen in isolation in man. Individuals under chronic stress often have co-existing disorders which may themselves be associated with hypercortisolemia. Depression, for example, provides one link between stress and increased cortisol in some individuals, since depression is not only a result of life stressor but it also acts as a stressor in its own right. 
Similarly some of the metabolic syndromes characterized by hypertension, visceral obesity and insulin resistance may result in stress rather than be caused by stress - and indeed may not be associated with increasing HPA activity at all - as shown in a recent report by Jessop et al. [16] who found low cortisol in obese subjects. Another disorder with hypothesized HPA axis abnormality is fibromyalgia, which is associated with pain and increased stress secondary to disability. However, again the increased cortisol may be part of the disease process rather than a response to stress.

While the general assumption in disease - is that abnormalities observed in mean ACTH and cortisol levels reflect increased activity of $\mathrm{CRH}$ neurons in the hypothalamus, analyzing alterations in HPA pulsatility provides an alternative means to evaluate the regulation or dysregulation of the HPA axis. In a rodent model of arthritis that is associated with increased corticosterone secretion [27], this increase is associated with shorter interpulse intervals and more continuous occupation of MR and GR. On the other hand looking at depression in man, we find that both pulse frequency and amplitude are normal. In addition, ultradian and circadian rhythms are normal. There is, however, an increase in the "basal", non-pulsatile components of cortisol secretion, suggesting that the effect may reflect trophic effects of ACTH at the adrenal. This is a different profile to that which would be observed if the subject was overresponding to ongoing life stressors, with sporadic increases in pulse number. These data would suggest that increased sensitivity of the adrenal to ACTH contributes to the increased cortisol rather than increased $\mathrm{CRH}$ secretion. These changes in adrenal sensitivity may be centrally mediated via autonomic control from the paraventricular nucleus of the hypothalamus, or else reflect a previous period of HPA hyperactivity resulting in altered adrenal responses $[10,14,15]$.

\section{Conclusion}

In conclusion, wide variation in the cortisol response is observed among individuals in response to a stressor. This variability is so marked that in any one individual it is not always possible to distinguish a stress response from a spontaneously occurring pulse. Indeed, we could put forward a totally different hypothesis that rather than activating cortisol release as an independent variable, acute stressors simply "advance" a spontaneous cortisol pulse, thereby acting as a synchronizer or "zeitgeber" for the ultradian cortisol rhythm, whose effectiveness will depend on a number of variables that control the individual's endogenous rhythm. This would explain our need to define stress response by group data, and stimulate the need to re-think the assumptions underlying cause and effect of cortisol increases in hu- mans. Furthermore, pulsatility itself may communicate different information to the system than the steady state levels usually studied in basic studies of HPA regulation. These changing levels of cortisol may result in different occupation of MR and GR throughout the circadian rhythm. Pulsatility also results in the engagement of rate-sensitive feedback mechanisms as well as more traditional level sensitive mechanisms. Finally, pulsatility analysis enables us to examine multiple aspects of the control of the HPA axis, extending our understanding well beyond mean cortisol levels.

\section{Acknowledgments}

The authors acknowledge the support of MH 50030 (E.A.Y.), MH 01931 (E.A.Y.), M01-RR00042 to the University of Michigan G-CRC, and MH 52724 to J.A.

\section{References}

[1] M. Abe, E. Herzog, S. Yamakaze, M. Straume, H. Tei, Y. Sakakai, M. Menaker, G. Block, Circadian rhythm in isolated brain regions, J. Neuosci. 22 (2002) 360-368.

[2] M.H. Andrews, R.J. Windle, Y.M. Kershaw, S.A. Wood, S.L. Lightman, C.D. Ingram, Rapid negative feedback effects of glucocorticoids within the hypothalamo-pituitary-adrenal axis, in: Proceedings of the British Neuroscience Association, April, 2001.

[3] M.H. Andrews, Glucocorticoid regulation of basal and stressinduced hypothalamo-pituitary-adrenal axis function, Ph.D. Thesis, University of Bristol, UK, 2002.

[4] M.J. Bradbury, S.F. Akana, M.F. Dallman, Roles of type I and II corticosteroid receptors in regulation of basal activity in the hypothalamo-pituitary-adrenal axis during the diurnal trough and the peak: evidence for a nonadditive effect of combined receptor occupation, Endocrinology 134 (1994) 1286-1296.

[5] M. Carnes, B.M. Goodman, S.J. Lent, H. Vo, R. Jaeckels, Coincident plasma ACTH and corticosterone time series: comparisons between young and old rats, Exp. Gerontol. 29 (1994) 625-643.

[6] M. Carnes, B.M. Goodman, S.J. Lent, H. Vo, High intensity venous sampling reveals subtle alterations in plasma adrenocorticotropin patterns in old rats, Endocrinology 133 (1993) 608-616.

[7] H.S. Chowdrey, P.J. Larsen, M.S. Harbuz, D.S. Jessop, G. Aguilera, D.J. Eckland, S.L. Lightman, Evidence for arginine vasopressin as the primary activator of the HPA axis during adjuvant-induced arthritis, Brit. J. Pharmacol. 116 (1995) 2417 2424.

[8] E.R. De Kloet, Brain corticosteroid receptor balance and homeostatic control, Front. Neuroendocrinol. 2 (1991) 95-164.

[9] E.R. DeKloet, G. Wallach, B.S. McEwen, Differences in corticosterone and dexamethasone binding to rat brain and pituitary, Endocrinology 95 (1975) 598-605.

[10] W.C. Engeland, D.S. Gann, Splanchnic nerve stimulation modulates steroid secretion in hypophysectomized dogs, Neuroendocrinology 50 (1989) 124-131.

[11] M. Follenius, C. Simon, G. Bradenberger, P. Lenzi, Ultradian plasma corticotropin and cortisol rhythms: time series analyses, J. Clin. Invest. 10 (1987) 261-266.

[12] E.S. Hanson, M.J. Bradbury, S.F. Akana, K.S. Scribner, A.M. Strack, M.F. Dallman, The diurnal rhythm in adrenocorticotro- 
pin responses to restraint in adrenalectomized rats is determined by caloric intake, Endocrinology 134 (1994) 2214-2220.

[13] A. Iranmanesh, G. Lizarralde, J.D. Veldhuis, Coordinate activation of the corticotropic axis by insulin-induced hypoglycemia: simultaneous estimates of beta-endorphin, adrenocorticotropin and cortisol secretion and disappearance in normal men, Acta Endocrinol. (Copenh.) 128 (1993) 521-528.

[14] M.S. Jasper, W.C. Engeland, Splanchnic neural activity modulates ultradian and circadian rhythms in adrenocortical secretion in awake rats, Neuroendocrinology 59 (1994) 97-109.

[15] M.S. Jasper, W.C. Engeland, Splanchnicotomy increases adrenal sensitivity to ACTH in nonstressed rats, Am. J. Physiol. 273 (1997) E363-E368.

[16] D.S. Jessop, M.F. Dallman, D. Fleming, S.L. Lightman, Resistance to glucocorticoid feedback in obesity, J. Clin. Endocrinol. Metab. 86 (2001) 4109-4114.

[17] D. Julian, R.J. Windle, S.A. Wood, Y.M. Kershaw, S.L. Lightman, C.D. Ingram, Basal and stress-induced hypothalamopituitary-adrenal (HPA) activity in aged male and female rats, in: Proceedings of the Anatomical Society, Symposium on Ageing and Longevity, London, September, 1999.

[18] A.M. Karssen, O.C. Meijer, I.C. van der Sandt, P.J. Lucassen, E.C. de Lange, A.G. de Boer, E.R. de Kloet, Multidrug resistance P-glycoprotein hampers the access of cortisol but not of corticosterone to mouse and human brain, Endocrinology 142 (2001) 2686-9416.

[19] C. Kirschbaum, K.M. Pirke, D.H. Hellhammer, The "Trier Social Stress Test"- a tool for investigating psychobiological stress responses in a laboratory setting, Neuropsychobiology 28 (1993) 76-81.

[20] D.T. Krieger, Rhythms in CRH, ACTH and corticosteroids, Endocr. Rev. 1 (1979) 123.

[21] S.L. Lightman, R.J. Windle, A.P. Da Costa, N. Shanks, C.D. Ingram, Lactation: a physiological model of stress hyporesponsiveness of the neuroendocrine system, in: A. Levy, E. Grauer, D. Ben Nathan, E.R. DeKloet (Eds.), New Frontiers in Stress Research, Harwood Academic Publishers, JMBH, The Netherlands, 1998, pp. 59-71.

[22] X.M. Ma, S.L. Lightman, G. Aguilera, Vasopressin and corticotropin-releasing hormone gene responses to novel stress in rats adapted to repeated restraint, Endocrinology 140 (1999) 36233632.

[23] J.M. Reul, F.R. van den Bosch, E.R. de Kloet, Differential response of type I and type II corticosteroid receptors to changes in plasma steroid level and circadian rhythmicity, Neuroendocrinology 45 (1987) 407-412.

[24] N. Shanks, R.J. Windle, P.A. Perks, M.S. Harbuz, D.S. Jessop, C.D. Ingram, S.L. Lightman, Early-life exposure to endotoxin alters hypothalamic-pituitary-adrenal function and predisposition to inflammation, Proc. Natl. Acad. Sci. 97 (2000) 5645-5650.

[25] R.L. Spencer, E.A. Young, P.H. Choo, B.S. McEwen, Glucocorticoid Type I and Type II receptor binding: estimates of in vivo receptor number, occupancy and activation with varying levels of steroid, Brain Res. 514 (1990) 37-48.

[26] E. van Cauter, R. Leproult, D.J. Kupfer, Effects of gender and age on the levels and circadian rhythmicity of plasma cortisol, J. Clin. Endocrinol. Metab. 81 (1996) 2468-2473.

[27] R.J. Windle, S.A. Wood, Y.M. Kershaw, S.L. Lightman, C.D. Ingram, M.S. Harbuz, Increased corticosterone pulse frequency during adjuvant-induced arthritis and its relationship to alterations in stress responsiveness, J. Neuroendocrinol. 13 (2001) 905911.

[28] R.J. Windle, S.A. Wood, N. Shanks, S.L. Lightman, C.D. Ingram, Ultradian rhythm of basal corticosterone release in the female rat: dynamic interaction with the response to acute stress, Endocrinology 139 (1998) 443-450.

[29] R.J. Windle, S.A. Wood, Y.M. Kershaw, S.L. Lightman, C.D. Ingram, M.S. Harbuz, Increased corticosterone pulse frequency during adjuvant-induced arthritis and its relationship to alterations in stress responsiveness, J. Neuroendocrinol. 13 (2001) 905911.

[30] R.J. Windle, S.A. Wood, S.L. Lightman, C.D. Ingram, The pulsatile characteristics of hypothalamo-pituitary-adrenal activity in female Lewis and Fischer 344 rats and its relationship to differential stress responses, Endocrinology 139 (1998) 4044-4052.

[31] E.A. Young, S.P. Kwak, J. Kottak, Negative feedback regulation following administration of chronic exogenous corticosterone, J. Neuroendocrinol. 7 (1995) 37-45.

[32] E.A. Young, J.F. Lopez, V. Murphy-Weinberg, S.J. Watson, H. Akil, The role of mineralocorticoid receptors in HPA axis regulation in humans, J. Clin. Endocrinol. Metab. 83 (1998) 3339-3345.

[33] E.A. Young, N.E. Carlson, M.B. Brown, 24-h ACTH and cortisol pulsatility in depressed women, Neuropsychpoharmacology 25 (2001) 267-276.

[34] E.A. Young, R.F. Haskett, S.J. Watson, H. Akil, Loss of glucocorticoid fast feedback in depression, Arch. Gen. Psychiatry 48 (1991) 693-699. 